\title{
Perfil epidemiológico das parasitoses intestinais em moradores de uma comunidade da Ilha de Boipeba, Bahia, Brasil
}

\author{
Epidemiological profile of intestinal parasites in residents of a community on the island \\ of Boipeba, Bahia, Brazil
}

\author{
Maria Eduarda Maia Vilar ${ }^{1 *}$, Nilo Manoe' Pereira Vieira Barreto², Neci Matos Soares ${ }^{3}$, Márcia Cristina \\ Aquino Teixeira ${ }^{4}$, Carlos Maurício Cardeal Mendes ${ }^{5}$
}

\begin{abstract}
${ }^{1}$ Enfermeira, Mestre em Processos Interativos dos Órgãos e Sistemas (UFBA); ${ }^{2}$ Mestre em Processos Interativos dos Órgãos e Sistemas (UFBA), Professor na Faculdade de Gestão e Negócios (FGN); ${ }^{3}$ Farmacêutica, Doutora em Biologia Celular e Molecular, Professora Titular da Faculdade de Farmácia (UFBA); ${ }^{4}$ Farmacêutica, Doutora em Biologia

Celular e Molecular, Professora Associada da Faculdade de Farmácia (UFBA); ${ }^{5}$ Médico, Doutor em Saúde Coletiva, Professor do Programa de Pós-graduação em Processos Interativos dos Órgãos e Sistemas (UFBA)
\end{abstract}

\begin{abstract}
Resumo
Introdução: as parasitoses intestinais constituem-se um importante problema de saúde pública mundial. Estas infecções são mais prevalentes em regiões tropicais impactando na morbimortalidade e aumento nos custos para o sistema de saúde. Objetivo: avaliar a prevalência das enteroparasitoses e sua associação com as condições socioeconômicas, sanitárias, ambientais e hábitos de vida em uma comunidade costeira do Nordeste brasileiro. Metodologia: estudo epidemiológico, descritivo e transversal, realizado de modo não probabilístico entre março a junho de 2017, com 105 moradores da llha de Boipeba, localizada no Sul da Bahia. O exame parasitológico de fezes foi realizado pelos métodos de sedimentação espontânea, Baermann-Moraes e FAUST. Um questionário foi aplicado para avaliar o perfil sociodemográfico da população. Resultados: do total de indivíduos avaliados, 52,4\% eram do sexo feminino e 57,1\% tinham entre 15 a 59 anos. Todos os indivíduos possuíam água encanada, porém não tratada, enquanto 91,4\% referiu ingerir vegetais crus e $45,7 \%$ não higienizavam as mãos antes as refeições. Sintomas gastrointestinais foram relatados em $82,8 \%$ dos indivíduos e infecções enteroparasitárias foram diagnosticadas em $69,6 \%$. Os parasitos mais frequentemente encontrados foram Ancilostomídeo (18,1\%) e Entamoeba coli $(43,8 \%)$. O principal fator de risco potencial para contrair a infecção por ancilostomídeos foi a não existência de poço artesiano na residência $(\mathrm{RP}=4,35)$, enquanto para Trichuris trichiura foi não dispor de pia no banheiro $(\mathrm{RP}=3,82)$. Conclusão: a comunidade analisada apresentou elevada prevalência de enteroparasitoses. Os hábitos precários de higiene e de acesso à água tratada, associados às condições ambientais e climáticas do local, podem ter contribuído para a elevada transmissão de geohelmintos observada.

Palavras-chave: Epidemiologia. Infecções Parasitárias. Saneamento. Comunidade Costeira
\end{abstract}

\begin{abstract}
Introduction: Intestinal parasitic infections are an important public health problem worldwide. They are more prevalent in tropical regions impacting in morbidity and mortality and costs for the health system. Objective: To evaluate the prevalence of enteroparasitosis and its association with socioeconomic, sanitary and environmental conditions and lifestyle habits in a coastal community in the Northeast Brazil. Methodology: This is an epidemiological, descriptive and cross-sectional study, carried out in a non-probabilistic manner between March and June 2017, with 105 residents of Boipeba Island in the Southern Bahia. The parasitological examination of feces was carried out by the methods of spontaneous sedimentation, Baermann-Moraes and FAUST. A questionnaire was applied to assess the sociodemographic profile of the population. Results: Of the total of individuals evaluated, $52.4 \%$ were female and $57.1 \%$ were between 15 and 59 years old. All individuals had piped, but untreated water, while $91.4 \%$ reported eating raw vegetables and $45.7 \%$ did not wash their hands before meals. Gastrointestinal symptoms were observed in $82.8 \%$ individuals and $69.6 \%$ were diagnosed with enteroparasitic infections. The most frequent parasites were the hookworm (18.1\%) and Entamoeba coli (43.8\%). The main potential risk factor for hookworm infection was the non-existence of an artesian well in the residence $(P R=4.35)$ and Trichuris trichiura it was not having a sink in the toilet ( $P R=3.82)$. Conclusion: The analyzed community has a high prevalence of enteroparasitosis, in addition to environmental and climatic conditions that contribute to the transmission of these infections.
\end{abstract}

Keywords: Epidemiology. Intestinal Diseases. Parasitic infections. Community. Coast

\section{INTRODUÇÃO}

As infecções parasitárias intestinais são consideradas doenças tropicais negligenciadas listadas pela OMS,

Correspondente/Corresponding: *Maria Eduarda Maia Vilar-End: Rua Basílio da Gama, s/n, Canela, Salvador-BA - Tel: (71) 3283-7373 - E-mail: vilar.eduarda@gmail.com representando a quarta principal causa de doenças transmissíveis e constituindo cerca de 46 a 57 milhões de anos de vida perdidos, ajustados por incapacidade (DAILY) ${ }^{1,2}$. Essas doenças são comuns em indivíduos que apresentam vulnerabilidade social, causando estigma e discriminação, além de ter um importante impacto na 
morbimortalidade e nos custos para o sistema de saúde. Contudo, são passíveis de medidas de controle e prevenção com baixo custo ${ }^{3}$.

De acordo com a Organização Mundial de Saúde 4 estima-se que no mundo 820 milhões de indivíduos estão infectadas por Ascaris lumbricoides, 460 milhões por Trichuris trichiura e 440 milhões por Ancilostomídeos 5 . Além disso, relatos apontam a existência de cerca de 370 milhões infectados por Strongyloides stercoralis, 500 milhões por Entamoeba histolytica e 280 milhões por Giardia duodenalis ${ }^{6,7}$. Estas infecções são mais prevalentes em regiões tropicais, afetando principalmente crianças em idade escolar e pré-escolar, acarretando deficiências nutricionais, atraso no crescimento e no desempenho escolar ${ }^{8,9}$, constituindo-se em um importante problema de saúde pública ${ }^{10}$. No Brasil, as condições climáticas ideais como alta temperatura e humidade ${ }^{11}$, associadas às condições sanitárias desfavoráveis, baixo nível de higiene e às deficiências nutricionais, principalmente em crianças ${ }^{12}$, entre outros, contribuem para alta prevalência destes parasitos no país ${ }^{12,13}$. Dentre os fatores de risco associados à transmissão de parasitoses, o saneamento básico e o acesso a água tratada são os mais relevantes. A associação fossa-poço é comum, aumentando os riscos de proliferação de doenças e parasitas por meio da contaminação da água subterrânea, principalmente em áreas rurais, onde o uso de fossas sépticas tem sido considerado uma conduta apropriada e relativamente barata ${ }^{7,8}$.

A transmissão de parasitos intestinais ocorre predominantemente por via fecal-oral, tanto pela ingestão de água como pelo consumo de alimentos contaminados com as formas infectantes dos parasitos, principalmente aqueles consumidos crus, como os vegetais e algumas carnes, bem como pela higienização incorreta das mãos, deixando resíduos fecais que são levados à boca ${ }^{14,15}$. Os diagnósticos laboratoriais das parasitoses são feitos principalmente usando exames parasitológicos de fezes ou por testes imunoenzimáticos ${ }^{16,17}$, enquanto o tratamento envolve a administração de medicamentos quimioterápicos, com alta eficiência e poucos efeitos colaterais. Mesmo com o avanço farmacológico, ainda não há uma droga satisfatória que seja de baixo custo, polivalente, que não tenha efeitos colaterais, adequada para uso em grávidas e crianças, de alta eficiência para todos os agentes, empregada em dose única e por via oral ${ }^{18,19}$.

Nesse contexto, o objetivo deste trabalho foi avaliar a prevalência das parasitoses intestinais e sua associação com os fatores socioeconômicos, sanitários, ambientais e hábitos de vida dos moradores de uma comunidade costeira turística do Nordeste brasileiro, que em virtude do patrimônio natural, a comunidade está inserida dentro de uma região reconhecida pela Organização das Nações Unidas para a Educação, a Ciência e a Cultura (UNESCO) como Reserva da Biosfera e Patrimônio da Humanidade.

\section{METODOLOGIA}

\section{Tipo de estudo e caracterização da amostra}

Trata-se de um estudo epidemiológico, descritivo, transversal realizado no período de março a junho de 2017 com 105 residentes do povoado de Moreré, que possui população total de 400 habitantes ${ }^{20}$, situado na Ilha de Boipeba, município de Cairú, Bahia, Brasil. A ilha é composta por quatro povoados (Velha Boipeba, Moreré, São Sebastião e Monte Alegre), com um total de 3256 habitantes, cuja região foi reconhecida pela UNESCO como Reserva da Biosfera e Patrimônio da Humanidade. As principais atividades econômicas são o turismo, a pesca artesanal e o extrativismo vegetal ${ }^{21}$.

\section{Coleta de dados e amostragem}

Foram realizadas quatro visitas à comunidade de Moreré para apresentação do projeto à comunidade, distribuição dos coletores e coleta de fezes. Além disso, foi aplicado um questionário para avaliar as condições socioeconômicas, sanitárias e educacionais dos participantes. Foram considerados elegíveis para o estudo todos os indivíduos do povoado de Moreré que estavam presentes em uma das três visitas à comunidade para aplicação dos questionários e coleta das amostras de fezes frescas. $\mathrm{Na}$ primeira visita foram coletadas 37 amostras, na segunda 46 e na terceira 22, totalizando 105 amostras. Foram excluídos residentes temporários, menores de idade cujos progenitores não estivessem presentes e indivíduos que não entregaram amostras fecais.

\section{Diagnóstico parasitológico}

As amostras fecais frescas foram coletadas e transportadas em isopor até o setor de Parasitologia do Laboratório de Análises Clínicas e Toxicológicas da Faculdade de Farmácia da Universidade Federal da Bahia (LACTFAR/ UFBA) para processamento no mesmo dia. Uma amostra de fezes de cada indivíduo foi examinada por três diferentes métodos parasitológicos: sedimentação espontânea ${ }^{22}$, Baermann-Moraes ${ }^{23}$ e FAUST $^{24}$.

\section{Análise estatística}

Foram calculados os coeficientes de prevalência para diferentes parasitoses. Para obtenção direta das razões de prevalência brutas e ajustadas (RP) realizou-se o modelo linear generalizado por regressão log-binomial. As variáveis incluídas no modelo foram selecionadas a partir da maior frequência univariada e que permitissem suficiência numérica para os modelos tentativos. A manutenção das variáveis independentes no modelo deveu-se à um ponto de corte arbitrário maior do que $30,0 \%$ nas razões de prevalências ajustadas ${ }^{25}$. Devido ao plano amostral não probabilístico não se fez necessário o cálculo de estatísticas inferenciais para a decisão da manutenção de variáveis no modelo final1-326,27. A análise estatística foi realizada no pacote estatístico $R$ versão 3.4 para Ubuntu Linux ${ }^{28}$. 


\section{Aspectos éticos}

O estudo obedeceu às recomendações da Resolução CNS 466 de 12 de dezembro de 2012 para o desenvolvimento de pesquisa com seres humanos. Todos os indivíduos que concordaram em participar do estudo assinaram o Termo de Consentimento Livre e Esclarecido e para os menores de idade, o Termo de Assentimento Livre e Esclarecido. Os resultados dos exames foram entregues diretamente aos participantes, e quando necessário, foram orientados quanto ao tratamento. Este projeto foi submetido e aprovado pelo Comitê de Ética em Pesquisa do Instituto de Ciências da Saúde (ICS) da Universidade Federal da Bahia (UFBA) sob número CAAE: 61990016.4.0000.5662, com subsídio próprio.

Ao final do estudo, foi realizado uma atividade de educação em saúde sobre parasitoses intestinais e foram entregues os laudos com os respectivos resultados dos exames a cada participante da pesquisa de forma individualizada. Todos que necessitaram de tratamento para alguma parasitose receberam a medicação gratuitamente, prescrita pelo médico da equipe e todas as orientações necessárias ao tratamento e prevenção das parasitoses intestinais.

\section{RESULTADOS}

Participaram do estudo cerca de 26,3\% (105/400) da população da comunidade de Moreré, sendo $52,4 \%$ do sexo feminino. A média da idade foi de $25,7(18,6)$, com a maioria, 37,1\%, entre 0 a 14 anos. Quanto a ocupação dos participantes, $40,0 \%$ (42/105) eram estudantes e $16,2 \%$ referiram retirar seu sustento do mar, através da pesca $(9,5 \%)$ e coleta de marisco $(6,7 \%)$ (Tabela 1$)$.

Tabela 1 - Distribuição das características demográficas na amostra da população estuda (n=105), 2017.

\begin{tabular}{|c|c|}
\hline Variáveis & $\begin{array}{l}\text { Total } \\
\text { n (\%) }\end{array}$ \\
\hline \multicolumn{2}{|l|}{ Sexo } \\
\hline Masculino & $50(47,6)$ \\
\hline Feminino & $55(52,4)$ \\
\hline \multicolumn{2}{|l|}{ Idade } \\
\hline 0-14 & $39(37,1)$ \\
\hline $15-29$ & $26(24,8)$ \\
\hline $30-44$ & $20(19,0)$ \\
\hline $45-59$ & $14(13,4)$ \\
\hline 60 e mais & $6(5,7)$ \\
\hline Média e Desvio padrão (anos) & $25,7(18,6)$ \\
\hline \multicolumn{2}{|l|}{ Ocupação } \\
\hline Dona de casa & $11(10,5)$ \\
\hline Pescador & $10(9,5)$ \\
\hline Marisqueiro & $7(6,7)$ \\
\hline Estudante & $42(40,0)$ \\
\hline Caseiro & $4(3,8)$ \\
\hline Motorista & $2(1,9)$ \\
\hline Manipulador de alimentos & $5(4,8)$ \\
\hline Serviços Gerais & $8(7,6)$ \\
\hline Outras & $16(15,2)$ \\
\hline
\end{tabular}

Fonte: Dados da pesquisa
Todos os indivíduos de Moreré (100\%) utilizavam fossa séptica e não tinham acesso a abastecimento de água tratada, sendo que $43,8 \%$ referiram a utilização de poços artesianos residenciais. Quanto ao estudo das variáveis relacionadas com a transmissão de parasitoses, $91,4 \%$ referiram ingerir vegetais crus e 38,1\% referiram comer carne mal passada. Cerca de $45,7 \%$ referiu não lavar as mãos antes das refeições e $24,8 \%$ ou após utilizar o vaso sanitário. O hábito de defecar no ambiente foi relatado por $41,9 \%$ dos participantes e todos possuíam hábitos de andar descalços em algum momento do dia. A maioria, 82,8\% referiu ter algum sintoma relacionado a parasitoses, sendo que $50,5 \%$ queixou-se de dor abdominal (Tabela 2).

Tabela 2 - Distribuição dos hábitos alimentares e de higiene, condições sanitárias e sintomas relacionadas às parasitoses na amostra da população estudada (n=105), 2017.

\begin{tabular}{|c|c|}
\hline Variáveis & $\begin{array}{l}\text { Total } \\
\mathrm{n}(\%)\end{array}$ \\
\hline \multicolumn{2}{|l|}{ Ingestão Alimento Cru ou Mal Passado } \\
\hline Carne bovina, suína, aves & $40(38,1)$ \\
\hline Peixe & $7(6,7)$ \\
\hline Marisco & $29(27,6)$ \\
\hline Ovo & $15(14,3)$ \\
\hline Vegetais & $96(91,4)$ \\
\hline \multicolumn{2}{|l|}{ Condições de Higiene Pessoal } \\
\hline Lavar as mãos antes das refeições & $57(54,3)$ \\
\hline Lavar as mãos após uso sanitário & $79(75,2)$ \\
\hline Hábito lavar frutas e verduras & $94(89,5)$ \\
\hline Hábito defecar no meio ambiente & $44(41,9)$ \\
\hline Hábito de andar descalço & $105(100)$ \\
\hline \multicolumn{2}{|l|}{ Condições Sanitárias e de Moradia } \\
\hline Água encanada não tratada ${ }^{1}$ & $105(100)$ \\
\hline Poço artesiano ${ }^{1}$ & $46(43,8)$ \\
\hline Rede de esgoto ${ }^{2}$ & $0(0)$ \\
\hline Vaso sanitário & $105(100)$ \\
\hline Pia no sanitário ${ }^{3}$ & $81(77,1)$ \\
\hline Pavimentação & $0(0)$ \\
\hline \multicolumn{2}{|l|}{ Sintomas relacionados a parasitoses } \\
\hline Ausência & $18(17,1)$ \\
\hline Presença & $87(82,8)$ \\
\hline \multicolumn{2}{|l|}{ Sintomas específicos } \\
\hline Diarreia & $13(12,4)$ \\
\hline Dor abdominal & $53(50,5)$ \\
\hline Perda de peso & $35(33,3)$ \\
\hline Prurido anal noturno & $23(21,9)$ \\
\hline Anemia referida & $19(18,1)$ \\
\hline Ascaris nas fezes & $9(8,6)$ \\
\hline $\begin{array}{l}{ }^{1} \text { A diferença }(56,2 \%) \text { referiu utilizar po } \\
{ }^{2} \text { Utiliza fossa séptica sanitária como o }\end{array}$ & munitário \\
\hline \multicolumn{2}{|c|}{$\begin{array}{l}{ }^{3} \text { A diferença }(22,9 \%) \text { referiu utilizar a pia da cozinha para higienização } \\
\text { das mãos e roupas. }\end{array}$} \\
\hline Fonte: Dados da pesquisa & \\
\hline
\end{tabular}


A frequência da infecção por parasitos intestinais nos indivíduos estudados foi de $69,6 \%$, dos quais $32,4 \%$ eram monoparasitados e $37,2 \%$ poliparasitados. 0 helminto mais frequente foram os Ancilostomídeos com 18,1\%, seguido de Trichuris trichiura com 12,4\%. Quanto os protozoários houve uma elevada ocorrência dos não patogênicos como Entamoeba coli e Endolimax nana, com $43,8 \%$ e $32,4 \%$, respectivamente (Tabela 3 ).

Tabela 3 - Frequência e espécies de parasitos encontrados na amostra da população estudada ( $n=105), 2017$.

\begin{tabular}{|c|c|}
\hline & $\begin{array}{l}\text { Total } \\
\text { n (\%) }\end{array}$ \\
\hline Parasitismo & $73(69,6)$ \\
\hline Monoparasitados & $34(32,4)$ \\
\hline Poliparasitados & $39(37,2)$ \\
\hline \multicolumn{2}{|l|}{ Helmintos } \\
\hline Ancilostomídeos & $19(18,1)$ \\
\hline
\end{tabular}

Trichuris trichiura

Ascaris lumbricoides

$13(12,4)$

Hymenolepis nana

$5(4,8)$

Strongyloides stercoralis

$2(1,9)$

Schistosoma mansoni

$1(1,0)$

$1(1,0)$

$1(1,0)$

Enterobius vermicularis

$4(3,8)$

Giardia lamblia

Protozoários não Patogênicos

Entamoeba coli

$34(32,4)$

Endolimax nana

$46(43,8)$

lodamoeba butschlii

$3(2,9)$

Fonte: Dados da pesquisa

Posteriormente, foram analisados os fatores de risco para a infecção dos parasitos mais identificados na população. No modelo ajustado, o maior fator de risco potencial para a infecção por ancilostomídeos foi não possuir poço artesiano na residência $(\mathrm{RP}=4,35)$ (Tabela 4).

Tabela 4 - Razão de prevalência bruta (análise bivariada) e ajustada (modelo múltiplo de Poisson final) para a associação entre potenciais fatores de risco e positividade por ancilostomídeos na amostra da população estudada (n=105), 2017.

\begin{tabular}{|c|c|c|c|c|c|}
\hline Potencial fator de risco & Ancilóstomo - n(\%) & Ancilóstomo + n(\%) & RPB & $\beta_{i}^{*}$ & RPAj \\
\hline Sexo & - & - & - & 1,107 & - \\
\hline Feminino & $51(92,7)$ & $4(7,3)$ & 1 & & 1 \\
\hline Masculino & $35(70,0)$ & $15(30,0)$ & 4,11 & & 3,03 \\
\hline Idade & - & - & - & 0,720 & - \\
\hline$<=45$ & $69(80,2)$ & $17(19,8)$ & 1 & & 1 \\
\hline$>45$ & $17(89,5)$ & $2(10,5)$ & 0,53 & & 2,05 \\
\hline Lavar as mãos antes das refeições & - & - & - & 0,127 & - \\
\hline $\operatorname{Sim}$ & $51(87,9)$ & $7(12,1)$ & 1 & & 1 \\
\hline Não & $35(74,5)$ & $12(25,5)$ & 2,11 & & 1,13 \\
\hline Costuma lavar frutas e verduras & - & - & - & 0,520 & - \\
\hline Sim & $79(87,8)$ & $11(12,2)$ & 1 & & 1 \\
\hline Não & $7(46,7)$ & $8(53,3)$ & 4,37 & & 1,68 \\
\hline Poço artesiano próximo & - & - & - & 1,470 & - \\
\hline $\operatorname{Sim}$ & $43(93,5)$ & $3(6,5)$ & 1 & & 1 \\
\hline Não & $43(72,9)$ & $16(27,1)$ & 4,16 & & 4,35 \\
\hline
\end{tabular}

RPB: razão de Prevalência Bruta-análise bivariada.

RPAj: razão de Prevalência Ajustada para as demais variáveis no modelo - Critério de Informação de Akaike (AIC) = 94,6; desvio residual/graus de liberdade do desvio residual =0,434; média dos resíduos: 0,007, variância: 1,$17 ; R^{2}$ ajustado:0,343.

* $B_{i:}$ coeficiente angular do modelo de regressão de Poisson; $6:-0,4170$.

Modelo final: $\log (E($ anc $)$ ) $)=-0,4170+1,107\left(\right.$ sexo $\left._{i}\right)+0,720\left(\right.$ idade $\left._{i}\right)+0,856\left(\right.$ mar $\left._{i}\right)+0,127\left(\right.$ mãos $\left._{i}\right)+0,520\left(\right.$ fru $\left._{i}\right)+1,470$ (poço $)$

Fonte: Dados da pesquisa

No modelo ajustado para a Trichuris trichiura, o no sanitário ( $R P=3,82)($ Tabela 5). maior risco para contrair infecção foi não dispor de pia

Tabela 5 - Razão de prevalência bruta (análise bivariada) e ajustada (modelo múltiplo de Poisson final) para a associação entre potenciais fatores de risco e positividade por Trichuris trichiura na amostra da população estudada (n=105), 2017.

\begin{tabular}{|c|c|c|c|c|c|}
\hline Potencial fator de risco & $\begin{array}{l}\text { T. trichiura - } \\
\mathrm{n}(\%)\end{array}$ & $\begin{array}{l}\text { T. trichiura + } \\
\mathrm{n}(\%)\end{array}$ & RPB & $\beta_{i}^{*}$ & RPAj \\
\hline Sexo & - & - & - & 1,072 & - \\
\hline Masculino & $45(90,0)$ & $5(10,0)$ & 1 & & 1 \\
\hline Feminino & $47(85,5)$ & $8(14,5)$ & 1,45 & & 2,92 \\
\hline
\end{tabular}


Maria Eduarda Maia Vilar, ,

\begin{tabular}{|c|c|c|c|c|c|}
\hline Idade & - & - & - & 0,537 & - \\
\hline$>45$ & $18(94,7)$ & $1(5,3)$ & 1 & & 1 \\
\hline$<=45$ & $74(86,0)$ & $12(14,0)$ & 2,64 & & 1,71 \\
\hline Comer carne crua ou malpassada & - & - & - & 0,584 & - \\
\hline Não & $58(89,2)$ & $7(10,8)$ & 1 & & 1 \\
\hline Sim & $34(85,0)$ & $6(15,0)$ & 1,39 & & 1,79 \\
\hline Comer marisco cru & - & - & - & 0,523 & - \\
\hline Não & $69(90,8)$ & $7(9,2)$ & 1 & & 1 \\
\hline Sim & $23(79,3)$ & $6(20,7)$ & 2,25 & & 1,69 \\
\hline Lavar as mãos após ir ao sanitário & - & - & - & 0,650 & - \\
\hline Sim & $71(91,0)$ & $7(9,0)$ & 1 & & 1 \\
\hline Não & $21(77,8)$ & $6(22,2)$ & 2,45 & & 1,92 \\
\hline Costuma lavar frutas e verduras & - & - & - & 0,600 & - \\
\hline Sim & $81(90,0)$ & $9(10,0)$ & 1 & & 1 \\
\hline Não & $11(73,3)$ & $4(26,7)$ & 2,67 & & 1,82 \\
\hline Dispor de pia no sanitário & - & - & - & 1,339 & - \\
\hline Sim & $75(92,6)$ & $6(7,4)$ & 1 & & 1 \\
\hline Não & $17(70,8)$ & $7(29,2)$ & 3,95 & & 3,82 \\
\hline
\end{tabular}

RPB: razão de Prevalência Bruta-análise bivariada.

RPAj: razão de Prevalência Ajustada para as demais variáveis no modelo - Critério de Informação de Akaike (AIC) = 84,3; desvio residual/graus de liberdade do desvio residual =0,4362; média dos resíduos: 0,016, variância: 1,22; $R^{2}$ ajustado:0,2258.

$B_{i:}$ coeficiente angular do modelo de regressão de Poisson; $B_{0}:-4,500$.

Modelo final: $\log \left(E(\text { tri })_{i}\right)=-4,500+1,072\left(\right.$ sexo $\left._{i}\right)+0,537\left(\right.$ idade $\left._{i}\right)+0,584\left(\right.$ car $\left._{i}\right)+0,523\left(\right.$ mar $\left._{i}\right)+0,650\left(\right.$ mãos $\left._{i}\right)$.

Fonte: Dados da pesquisa

\section{DISCUSSÃO}

O município de Cairú é um dos dois únicos municípios brasileiros inteiramente arquipelágicos. A llha de Boipeba, na qual a comunidade está inserida, pertence a esta cidade. A ilha é limitada, na maior parte do seu território, pelo oceano Atlântico e ao sul pelo Rio dos Infernos, área $90 \mathrm{~km}^{2}$ com um perímetro de $37 \mathrm{Km}$. Apresenta vegetação típica da mata atlântica, assim como os ecossistemas de restingas, recifes-de-corais, manguezal e possui clima quente e úmido, tropical e costeiro ${ }^{29,30}$. Toda a ilha, assim como o arquipélago à qual pertence, foi declarada como Área de Proteção Ambiental (APA) ${ }^{30}$. O turismo, indústria do petróleo e pesca predatória são as principais atividades causadoras de conflitos e impactos negativos aos ecossistemas costeiros na APA. Os impactos decorrentes destas atividades podem promover a perda de biodiversidade, problemas de saneamento ambiental e ameaças à saúde humana ${ }^{31,32}$. Segundo o Plano de Manejo da APA Tinharé-Boipeba e a pesquisa de campo, as residências nas margens dos rios nos distritos de Cairú, lançam esgotos in natura nos rios, estuários, ou no mar ${ }^{30,33}$. Tais ações podem representar fatores importantes para a aquisição e manutenção do ciclo de parasitos intestinais ${ }^{34}$.

Apesar de ser detentor de uma das menores populações dentre os povoados componentes do município de Cairú ${ }^{35}$, Moreré apresenta características demográficas, econômicas e sociais que não diferem do município, possuindo uma população majoritariamente de baixa renda. No estudo pôde-se observar que a maioria dos moradores $(91,4 \%)$ tinham o hábito de ingerir vegetais crus, comerem carnes de maneira geral mal passadas $(38,1 \%)$, não lavarem as mãos antes das refeições $(45,7 \%)$ ou após utilizarem o vaso sanitário $(24,8 \%)$, além de defecarem no ambiente $(41,9 \%)$ e todos referirem andar descalços (100\%). Todos esses hábitos precários de higiene, associados a um saneamento básico ineficiente, favorecem a contaminação do solo e das fontes de água, mantendo a prevalência destas parasitoses na região ${ }^{36}$. Além disso, $22,9 \%$ dos indivíduos não possuíam pia dentro do banheiro e referiram usar a pia da cozinha ou da lavanderia para lavagem das mãos após o uso do sanitário, favorecendo a contaminação dos alimentos e de utensílios da cozinha por parasitos e coliformes fecais. Essas condições reunidas favorecem a principal forma de contaminação, fecal-oral, bem como a manutenção de parasitoses na população estudada.

Outra informação relevante é que alguns habitantes utilizavam poços construídos com manilhas dentro de suas propriedades, com o objetivo de diminuir os custos com o consumo de água. Entretanto, a perfuração desses poços com locação inadequada e sem obedecer a critérios técnicos, coloca em risco a qualidade da água de consumo, tornando-os mais suscetíveis à contaminação por águas superficiais ou dejetos de fossas sépticas, sendo, portanto, um perigo para a saúde humana ${ }^{37}$. A perfuração de poços artesianos deve ser preferencialmente em locais altos e livres de inundações, com distância mínima de 15 metros de fossas sépticas e 45 metros de qualquer outra fonte de contaminação, como cemitérios, lixões, galerias de infiltrações, dentre outros ${ }^{38}$.

A fossa séptica, quando não há tratamento de água e esgoto, é a opção mais comum em para receber os re- 
síduos e dejetos residenciais e quando bem construídas e afastadas de cursos d'água, não oferecem riscos de contaminação. Neste estudo, $100 \%$ dos participantes da pesquisa utilizavam fossas sépticas e $43,8 \%$ utilizavam água de poços artesianos residenciais para consumo. Considerando o tamanho pequeno do terreno das casas e a região ter baixo relevo e alto índice pluviométrico, é possível a contaminação de poços por dejetos das fossas ou águas superficiais. Além disso, a manipulação de água dos poços com as mãos sujas, pode também ter contribuído para a contaminação da água de consumo e consequentemente, para a elevada frequência de parasitoses observada no povoado.

A distribuição por idade da população estudada apresentou uma frequência de indivíduos menores de 15 anos $(37,1 \%)$, caracterizando uma população bastante jovem. Isso pode ter refletido na ocupação mais frequentemente observada, a de estudante, o que correspondeu a $40,0 \%$ dos participantes. As atividades de pescador, marisqueiro e dona de casa foram as mais frequentes entre a população adulta $(26,7 \%)$, sendo um resultado esperado já que a pesca é uma das principais atividades econômicas do povoado ${ }^{21}$.

Neste estudo, 69,6\% dos participantes apresentaram resultados positivos nos exames parasitológicos. Estudos no Brasil entre populações adultas e de crianças, demonstraram frequências semelhantes à encontrada, como, por exemplo, entre escolares na região metropolitana de Salvador, com $78,9 \%$ de positividade, sendo os comensais Entamoeba coli e Endolimax nana os mais frequentes ${ }^{39}$; em áreas de assentamento de Sergipe, com $69,4 \%$ das amostras positivas para Endolimax nana ${ }^{40} \mathrm{e}$ em Eirunepé-AM, com $64,4 \%$ de positividade para Entamoeba coli ${ }^{11}$. Estes protozoários não são patogênicos e a sua alta frequência é considerado um importante indicador epidemiológico, pois reflete as condições sanitárias, higiênicas e socioeconômicas às quais as populações estão expostas $^{39}$. Entre os helmintos, o Ascaris lumbricoides e o Trichuris trichiura, foram os mais frequentes nos três estudos acima. Por outro lado, na região metropolitana do Rio de Janeiro, em um estudo englobando diversos bairros da cidade, encontrou $17,5 \%$ das amostras positivas para pelo menos um parasita, porém os protozoários patogênicos, configuraram como os mais frequentes ${ }^{42}$.

A alta prevalência de Ancilostomídeos em Moreré $(18,1 \%)$, sugere uma endemicidade para este parasito na região. Condições ambientais (solo arenoso, rico em matéria orgânica, clima quente e úmido), associadas a condições de saneamento básico deficiente (ausência de esgotamento sanitário e de água potável para o consumo) e hábito de defecar no meio ambiente, podem favorecer a transmissão do parasito. As mesmas condições que favorecem a prevalência de ancilostomídeos na região são ideais para os outros geohelmintos também encontrados. Entretanto, a frequência relativamente baixa de Ascaris lumbricoides em nosso estudo (4,8\%), sugere que outras variáveis, como o hábito de andar descalço no solo, pelo menos em algum momento do dia, relatado por todos os moradores, contribuem para a manutenção da ancilostomose na população, além dos outros fatores descritos acima.

No modelo final ajustado, o perfil sexo masculino, idade acima de 45 anos, não lavar as mãos antes das refeições, não lavar frutas e verduras e não possuir poço artesiano, apresentou a maior frequência para contrair a infecção por ancilostomídeos, com $62,5 \%$ de positividade. Esse perfil corresponde com a literatura, geralmente pela maior exposição ao solo dos indivíduos durante suas atividades laborais, bem como hábitos de levar alimentos ou mãos não higienizadas a boca que pode propiciar, de forma menos usual, a penetração das larvas filarióides dos ancilostomídeos na mucosa oral-esofágica ${ }^{42,43}$. De acordo com as análises de prevalência bruta e ajustada, o maior risco para contrair infecção por ancilostomídeos foi a não existência de poço artesiano na residência $(R P=4,35)$. Considerando que a principal forma de infecção por Ancilostomídeos é a penetração por via cutânea, a ausência de poço artesiano como principal fator de prevalência poderia ser justificada pela necessidade dos moradores de se deslocarem a pé com maior frequência para as regiões vizinhas em busca da água. .

A partir do modelo final ajustado para a infecção por Trichuris trichiura, o perfil sexo feminino, idade menor que 45 anos, não lavar frutas e verduras e não dispor de pia no sanitário, apresentou a maior risco para a infecção pelo helminto. Esse perfil corresponde ao relatado na literatura, sendo a trichuríase mais frequente em crianças e adultos jovens de populações pobres vulneráveis ${ }^{9,36,44}$. Estudos no Brasi| ${ }^{37,39,42,45,46}$ e na África ${ }^{47,48}$, ressaltam a importância do saneamento e controle da qualidade da água, em especial para ingestão, na prevenção de doenças, principalmente parasitoses intestinais.

\section{CONCLUSÃO}

Neste estudo foi possível observar à elevada prevalência da infecção por parasitos intestinais em moradores da comunidade de Moreré, na llha de Boipeba, ao sul da Bahia. Os parasitos patogênicos mais frequentemente encontrados foram os Ancilostomídeos e Trichuris trichiura. As condições ambientais e climáticas favoráveis da região, associadas as precárias condições sanitárias e falta de tratamento da água, podem favorecer à transmissão desses geohelmintos e de outros parasitos entre os moradores. Políticas públicas são necessárias para melhorias na região, principalmente considerando que o povoado de Moreré está inserido em um polo turístico do estado e obtém royalties pela exploração de gás natural e petróleo na área marítima do município. Investimentos em infraestrutura sanitária, bem como em educação, irão refletir diretamente na sua saúde da população. 


\section{REFERÊNCIAS}

1. LAURSEN, L. Tropical disease: a neglected cause. Nature, [s.I.], v. 533, n. 7602, p. 68-69, May 2016. DOI: 10.1038/533s68a.

2. FELEKE, B. E. Nutritional Status and Intestinal Parasite in School Age Children: a comparative cross-sectional study. Int. J. Pediatr., [s.I.], v. 2016, p. 1-8, 2016. DOI: 10.1155/2016/1962128.

3. BAHK, Y. Y. et al. Prevention and Control Strategies for Parasitic Infections in the Korea Centers for Disease Control and Prevention. Korean J. Parasitol., Seoul, v. 56, n. 5, p. 401-408, 31 Oct. 2018. DOI: 10.3347/kjp.2018.56.5.401.

4. WORLD HEALTH ORGANIZATION (WHO). Soil-transmitted helminth Infections. Geneva: WHO, 2020. Disponível em: https://www.who.int/ news-room/fact-sheets/detail/soil-transmitted-helminth-infections. Acesso em: 5 May 2020.

5. CENTERS FOR DISEASE CONTROL AND PREVENCION (CDC). ParasitesSoil-Transmitted Helminths. U.S. department of Health \& Human Services: USA. Disponível em: https://www.cdc.gov/parasites/sth/ index.html. Acesso em: 26 May 2020.

6. GARCíA, C. G.; et al. Drug Resistance Mechanisms in Entamoeba histolytica, Giardia lamblia, Trichomonas vaginalis, and Opportunistic Anaerobic Protozoa. Antimicrobial Drug Resistance, [s.I.], p. 613-628, 2017. DOI: 10.1007/978-3-319-46718-4_40.

7. FONSECA, E. O. L. et al . Prevalência e fatores associados às geohelmintíases em crianças residentes em municípios com baixo IDH no Norte e Nordeste brasileiros. Cad. Saúde Pública, Rio de Janeiro, v. 26, n. 1, p. 143-152, Jan. 2010 . Disponível em: http://www.scielo. br/scielo.php?script=sci_arttext\&pid=S0102-311X2010000100015\& Ing=en\&nrm=iso. Acesso em: 22 jun. 2020. DOI:10.1590/S0102$311 \times 2010000100015$.

8. COOPER, P. J. et al. Impact of early life exposures to geohelminth infections on the development of vaccine immunity, allergic sensitization, and allergic inflammatory diseases in children living in tropical Ecuador: the ecuavida birth cohort study. BMC Infect. Dis., London, v. 11, n. 1, p. 184-200, 29 June 2011. DOI: 10.1186/1471-2334-11-184.

9. BETHONY, J. et al. Soil-transmitted helminth infections: ascariasis, trichuriasis, and hookworm. Lancet, London, v. 367, n. 9521, p. 15211532, May 2006. DOI: 10.1016/s0140-6736(06)68653-4.

10. BRASIL. MINISTÉRIO DA SAÚDE. Guia Prático para o Controle das Geo-helmintíases Brasília: Ministério da Saúde, 2018. 33 p. Disponível em: https://bvsms.saude.gov.br/bvs/publicacoes/guia_pratico_ controle_geohelmintiases.pdf. Acesso em: 22 jun. 2020.

11. SANTOS, D. E.; WIEBbelLING, A. M. P.; MeZZARI, A. Parasitos intestinais: aspectos gerais e prevalência em uma escola da periferia de Porto Alegre-RS. Newslab, [s.I], v. 60, p. 118-34, 2003.

12. CARILLO, M. R. G. G.; LIMA, A. A.; NICOLATO, R. C. L. Prevalência de enteroparasitoses em escolares do bairro Morro de Santana no município de Ouro Preto, MG. Rev. Bras. de Análises Clínicas, Rio de Janeiro, v. 37, n. 3, p. 191-93, 2005.

13. FREI, F.; JUNCANSEN, C.; RIBEIRO-PAES, J. T. Levantamento epidemiológico das parasitoses intestinais: viés analítico decorrente do tratamento profilático. Cad. Saúde Pública, Rio de Janeiro, v. 24, n. 12, p. 2919-2925, dez. 2008. DOI: 10.1590/s0102-311×2008001200021.

14. EFSTRATIOU, A.; ONGERTH, J. E.; KARANIS, P. Waterborne transmission of protozoan parasites: review of worldwide outbreaks an update 2011国2016. Wat. Res., Oxford,, v. 114, p. 14-22, May 2017. DOI: 10.1016/j.watres.2017.01.036.

15. SILVA, S. K. M. et al. TAENIA SP.: importância da higienização como forma de profilaxia. Mostra Científica da Farmácia, Quixabá, v. 4, n. 2, Aug. 2018. Disponível em: http://publicacoesacademicas. unicatolicaquixada.edu.br/index.php/mostracientificafarmacia/article/ view/2354/1913. Acesso em: 16 jun. 2020. ISSN: 2358-9124.

16. LACERDA, J. da Silva; DIAS, M. E. Deteç̧ão de Giardia lamblia em Exames Parasitológicos de Fezes: Avaliação Comparativa de Resultados em Laboratório de Análises Clínicas de Rede Privada e Pública. Revista Saúde UniToledo, São Paulo, v. 01, n. 01, p. 147-156, 2017. Disponível em: http://ojs.toledo.br/index.php/saude/article/view/2444. Acesso em: 22 jun. 2020.

17. CUNHA, R. A.; LEVENHAGEN, M. A.; COSTA-CRUZ, J. M. Elisa/ Fotoluminescência de quantum dots no diagnóstico sorológico da estrongiloidíase humana. Investigação, França, v. 15, n. 6, 2016. Disponível em: DOI: https://doi.org/10.26843/investigacao.v15i6.1512. Acesso em: 16 jun. 2020.

18. CHEHTER. L.; CABEÇA, M. Parasitoses intestinais. RBM Rev. Bras. Med., São Paulo, v. 57, p. 225-226, nov. 2000. Disponível em: https:// pesquisa.bvsalud.org/portal/resource/pt/lil-283936. Acesso em: 20 jun. 2020.

19. DOS SANTOS, M. M.; PAVANELLI, M. F. Fatores associados a compra de antiparasitários sem apresentação de receita médica em clientes de uma farmácia de Campo Mourão, PR. SaBios, Campo Mourão, v. 11, n. 2, p. 07-12, mar. 2017. Disponível em: http://revista2.grupointegrado. br/revista/index.php/sabios2/article/view/1809. Acesso em: 20 jun. 2020. ISSN: 1980-0002.

20. MARTINS, L. A. et al. Promovendo a sustentabilidade em comunidades quilombolas e ribeirinhas. Adolesc. Saude, Rio de Janeiro, v. 12, n.1, p. 60-64, mar. 2015. Disponível em: http://www.adolescenciaesaude.com/ detalhe_artigo.asp?id=492. Acesso em: 22 jun. 2020.

21. PORTAL DA PREFEITURA MUNICIPAL DE CAIRÚ. Cairú-BA. Disponível em: http://cairu.ba.io.org.br/. Acesso em: 5 jun. 2017.

22. HOFFMANN, W. A.; PONS, J. A.; JANER, J. L. Sedimentation concentration method in schistosomiasis. Public Health, London, v.9, p. 283-298, 1934.

23. MORAES, R. G. Contribuição para o estudo do Strongyloides stercoralis e da estrongiloidíase no Brasil. Rev.Saúde Pública, Rio de Janeiro, v. 1, p. 507-624, 1948.

24. FAUST, E. C. et al. A Critical Study of Clinical Laboratory Technics for the Diagnosis of Protozoan Cysts and Helminth Eggs in Feces. Am. J.Trop. Med. Hyg., Cleveland, v. 1-18, n. 2, p. 169-183, Mar. 1938. Disponível em: http://dx.doi.org/10.4269/ajtmh.1938.s1-18.169.

25. COUTINHO, L. M. S.; SCAZUFCA, M.; MENEZES, P. R. Métodos para estimar razão de prevalência em estudos de corte transversal. Rev. Saúde Pública, Rio de Janeiro, v. 42, n. 6, p. 992-998, dez. 2008. FapUNIFESP (SciELO). Disponível em: http://dx.doi.org/10.1590/s003489102008000600003 . Acesso em: 05 jun. 2020

26. LUDWIG, D. A. Use and misuse of p-values in designed and observational studies: guide for researchers and reviewers. Aviat. Space Environ. Med., Washington, v. 76, p. 675-780, July 2005.

27. WASSERSTEIN, R. L.; LAZAR, N. A. The ASA Statement on p-Values: context, process, and purpose. Am. Stat., Estados Unidos, v. 70, n. 2, p. 129-133, 2 Apr. 2016. DOI: 10.1080/00031305.2016.1154108.

28. $R$ DEVELOPMENT CORE TEAM. R: A language and environment for statistical computing. Vienna, Austria: R Foundation for Statistical Computing, 2017. Disponível em: http://www.R-project.org. Acesso em: 22 June 2020.

29. SUPERINTENDÊNCIA DE ESTUDOS ECONÔMICOS (SEI.) Cairu. In: Estatísticas dos Municípios Baianos. Salvador: SEI, 2014. V.4. P.37-55. 
30. GONÇALVES, V. P. et al. Gerenciamento Costeiro e Serviço Ecossistêmico de Qualidade das Águas das Ilhas deTinharé Boipeba, Cairu, Bahia (Brasil). In: Semana de Mobilização Científica-SEMOC, n.22, 2019, Salvador. Disponível em: http://ri.ucsal.br:8080/jspui/ bitstream/prefix/1368/1/Gerenciamento\%20costeiro\%20e\%20 servi\%C3\%A7o\%20ecossist\%C3\%AAmico\%20de\%20qualidade $\% 20$ das\%20\%C3\%A1guas\%20das\%20llhas\%20de\%20Tinhar\%C3\%A9\%20 e\%20Boipeba\%2C\%20Cairu\%2C\%20Bahia\%20\%28Brasil\%29.pdf. Acesso em: 05 jun. 2020

31. ELLIFF, C. I.; RUY, K. P K. “Ecosystem Services Provided by Coral Reefs In a Southwestern Atlantic Archipelago." Ocean Coast. Manag., Oxford, v. 136, p. 49-55. DOI: 10.1016/j.ocecoaman.2016.11.021.

32. PAIXAO, L. H.; ALENCAR, C. M. M. ; GONÇALVES, M. V. P. Dinâmica pesqueira de Gamboa no mundo rural costeiro do Município de CairuBA. In: SEMINÁRIO NACIONAL ESPAÇOS COSTEIROS: ESPAÇOS DE PRODUÇÃO, ESPAÇOS DE VIDA, 3., 2016, Salvador. Anais...Salvador, 2016.

33. INSTITUTO DO MEIO AMBIENTE E RECURSOS HÍDRICOS (INEMA). PlanodeManejoTinharéeBoipeba. Disponívelem: http://www.inema.ba.gov. br/gestao-2/unidades-de-conservacao/plano-de-manejo/plano-demanejo-tinhare-e-boipeba/. Acesso em: 05 jun. 2020.

34. RECH, S. C. et al. Frequência de enteroparasitas e condições socioeconômicas de escolares da cidade de São MarcosRS. Semina, Londrina, v. 37, n. 1, p. 25-32, 24 nov. 2016.DOI: 10.5433/1679-0367.2016v37n1p25.

35. BRASIL. Censo Demográfico 2010: Características da população e dos domicílios, resultados do universo. Rio de Janeiro: IBGE, 2011. Disponível em: http://www.ibge.gov.br/home/estatistica/ populacao/ censo2010/caracteristicas_da_populacao/ resultados_do_universo. pdf. Acesso em: 7 jun. 2017.

36. CARNEIRO, M.; ANTUNES, C. M. de F. Epidemiologia: introdução e conceito. In: NEVES, D.P. et al. (Eds). Parasitologia Humana. 13 ed. São Paulo: Atheneu, 2016. cap. 3. p.15-26.

37. RAZZOLINI, M. T. P.; GÜNTHER, W. M. R. Impactos na saúde das deficiências de acesso a água. Saúde e Soc., São Paulo, v. 17, n. 1, p. 21-32, mar. 2008. DOI: 10.1590/s0104-12902008000100003.

38. FUNDAÇÃO NACIONAL DE SAÚDE (FUNASA). Manual de orientaçoes técnicas para elaboração de propostas para o programa de melhorias sanitárias domiciliares - Funasa. Disponível em: http://www.funasa. gov.br/site/wpcontent/files_mf/manual_msd3_2.pdf. Acesso em: 26 nov. 2020.
39. SEIXAS, M. T. L. et al. Avaliação da frequência de parasitos intestinais e do estado nutricional em escolares de uma area periurbana de Salvador, Bahia, Brasil. Rev. Patol. Trop., Goiania, v. 40, n. 4, p. 304-314, 2 jan. 2012. DOI: $10.5216 /$ rpt.v40i4.16762.

40. OLIVEIRA, G. G. et al. Prevalence Of Intestinal Parasitoses In Families Of Landless Workers' Movement. Rev. Enferm. UFPE on line, Recife, v. 6, n. 10, p. 2490-2496, Oct, 2012. Disponível em: DOI: 10.5205/reuol.311124934-1-LE.0610201222

41. ARAUJO, C. F.; FERNÁNDEZ, C. L. Prevalência de parasitoses intestinais na cidade de Eirunepé, Amazonas. Rev. Soc. Bras. Med. Trop., Brasília, v. 38, n. 1, p. 69-69, fev. 2005. DOI: 10.1590/s0037-86822005000100016.

42. FARIA, C. P. et al. Geospatial distribution of intestinal parasitic infections in Rio de Janeiro (Brazil) and its association with social determinants. Plos Negl. Trop. Dis., San Francisco, v. 11, n. 3, p. 54-45, 8 Mar. 2017. DOI: 10.1371/journal.pntd.0005445.

43. ANTUNES, L. et al. Parasitos em hortaliças comercializadas no sul do Rio Grande do Sul, Brasil. Rev. Ciênc. Méd. Biol., Salvador, v. 12, n. 1, p. 45-49, 21 ago. 2013. DOI:10.9771/cmbio.v12i1.6543.

44. PRIETO-PÉREZ, L. et al. Formación médica continuada: Salud Internacional y atención al viajero Geohelmintos. Enferm. Infecc. Microbiol. Clín., Barcelona, v. 34, n. 6, p. 384-389, June 2016. DOI: 10.1016/j.eimc.2016.02.002.

45. MASCARINI, L. M. et al. Impacto de um Programa de Saneamento Ambiental na Prevalência e na Incidência das Parasitoses Intestinais na População de Idade Escolar de Salvador. Revista VeraCidade, Salvador, v. 4, A. 1, mar. 2009.

46. MORAES, L.R.S; CANCIO, J. A.; CAIRNCROSS, S. Impact of drainage and sewerage on intestinal nematode infections in poor urban areas in Salvador, Brazil. Trans. R. Soc. Trop. Med. Hyg., London, v. 98, n. 4, p. 197-204, Apr. 2004. Oxford University Press (OUP). http://dx.doi. org/10.1016/s0035-9203(03)00043-9.

47. YOUMBI, J. G. T. et al. Pollution de l'eau souterraine et risques sanitaires à Yaoundé au Cameroun. C. R. Biol., Paris, v. 336, n. 5-6, p. 310-316, May 2013. Disponível em: http://dx.doi.org/10.1016/j. crvi.2013.04.013. Acesso em: 20 Nov. 2020.

48. AMOAH, I. D. et al. Contribution of Wastewater Irrigation to Soil Transmitted Helminths Infection among Vegetable Farmers in Kumasi, Ghana. Plos Negl. Trop. Dis., San Francisco, v. 10, n. 12, p. 51-61, 6 Dec. 2016. DOI: 10.1371/journal.pntd.0005161.
Submetido em: 26/06/2020

Aceito em: 01/12/2020 